\title{
Plataforma Google Trends@: Análise do Interesse de Brasileiros na Área de Estética Dentária e Saúde Bucal
}

Google Trends ${ }^{\circledR}$ Platform: Analysis of Brazilian Interests in the Field of Dental Aesthetics and Oral Health

Plataforma Google Trends®: Análisis de Interés de los Brasileños en el Campo de la Estética Dental y la Salud Bucal

Bianca de Almeida CAPELLARI

Graduação em Odontologia pelo Centro Universitário Sagrado Coração - Unisagrado - USC, 17011-160 Bauru - SP, Brasil https://orcid.org/0000-0003-2725-4409

Fábio Silveira DE SOUZA

Doutor em Odontologia, Centro Universitário Sagrado Coração - Unisagrado - USC, 17011-160 Bauru - SP, Brasil https://orcid.org/0000-0002-6170-3426

Rhillari Kimberlli da Costa RIBEIRO

Aluna de Iniciação Científica - Ensino Médio - (PIBIC-EM) - Centro Universitário Sagrado Coração - Unisagrado - USC, 17011-160 Bauru - SP, Brasil https://orcid.org/0000-0002-3354-1239

Marina CARNEIRO

Aluna de Iniciação Científica - Ensino Médio - (PIBIC-EM) - Centro Universitário Sagrado Coração - Unisagrado - USC, 17011-160 Bauru - SP, Brasil https://orcid.org/0000-0003-0065-2044 Karin Cristina da SILVA MODENA

Doutora em Odontologia, Centro Universitário Sagrado Coração - Unisagrado - USC, 17011-160 Bauru - SP, Brasil https://orcid.org/0000-0002-0926-1253

Thiago Amadei PEGORARO

Doutor em Odontologia, Centro Universitário Sagrado Coração - Unisagrado - USC, 17011-160 Bauru - SP, Brasil https://orcid.org/0000-0003-2867-1239

Joel Ferreira SANTIAGO JUNIOR

Professor das disciplinas de graduação: Prótese Dentária e Implantodontia da Unisagrado (Bauru); Professor Contratado III (Doutor) junto ao departamento de Prótese e Periodontia, Faculdade de Odontologia de Bauru (USP) 17012-901 Bauru - SP, Brasil

https://orcid.org/0000-0003-1735-2224

\section{Resumo}

Introdução: Pesquisas em canais digitais sobre o interesse de brasileiros em diferentes áreas da saúde bucal e estética dentária são relevantes. Objetivo: Este estudo usou a plataforma de busca Google Trends $\AA$ (GT) a fim de realizar uma revisão para análise sobre dez tópicos importantes para a Odontologia no Brasil. Métodos: A busca foi organizada com palavras chaves relacionadas a estética dentária/facial (A) e saúde bucal (B). Os tópicos foram pesquisados individualmente por meio do índice de volume de pesquisa (IVP). Os dados quantitativos foram submetidos a teste de normalidade e adotado índice de significância de 0.05 . Resultados: Os principais resultados indicaram que, no grupo A, clareamento dentário é o termo mais analisado, apresentando diferença significativa na comparação com os demais termos: lente de contato dental, faceta dental, harmonização facial e preenchimento labial, $p<0,05$. Para o grupo B: saúde bucal e herpes oral foram os termos mais avaliados, não apresentando diferença significativa entre si, $p>0,05$. Destacou-se que houve um maior IVP para unitermos relacionados a estética dentária/face nos últimos anos (2016-2019), $\mathrm{p}<0,05$, todavia o Grupo B demonstrou menor variação (IVP) no mesmo período. Conclusão: Houve um aumento expressivo no uso de unitermos relacionados ao Grupo A nos últimos anos na plataforma GT e uma tendência de manutenção para os unitermos de saúde bucal. Torna-se importante que políticas de ensino em Odontologia contemplem o interesse da comunidade e valorizem a transmissão e conscientização de informações corretas em Odontologia para o desenvolvimento de uma boa prática de atendimento clínico na comunidade.

Descritores: Saúde Bucal; Estética; Odontologia.

\section{Abstract}

Introduction: Researchs on digital channels about the interest of brazilians in different areas of oral health and dental aesthetics are relevant. Objective: This study used Google Trends (c) (GT) plataform search to conduct a review to analyze ten important topics for dentistry in Brazil. The search was organized with keywords related to dental/facial aesthetics $(A)$ and oral health $(B)$. Methods: The topics were searched individually using the search volume index (SVI). Quantitative data were subjected to a normality test and a significance index of 0.05 was adopted. Results: The main results indicated that, in group A, tooth whitening is the most analyzed term, presenting a significant difference in comparison with the other terms: dental contact lens, dental facets (Ceramic Laminate Veneer), facial harmonization, and lip filling, $p<0.05$. For group B: oral health and Oral Herpes was the most evaluated terms, with no significant difference between them, $p>0.05$. It was highlighted that there was a higher SVI for terms related to dental aesthetics/face in recent years (2016-2019), $p<0.05$, however Group B showed less variation (SVI) in the same period. Conclusions: There has been a significant increase in the use of keywords related to Group $A$ in recent years at GT platform and a maintenance trend for oral health keywords. Teaching policies in Dentistry must contemplate the interest of the community and value the transmission and awareness of correct information in Dentistry for the development of good clinical care practice in the community.

Descriptors: Oral Health; Aesthetics; Dentistry.

\section{Resumen}

Introducción: Es relevante la investigación en canales digitales sobre el interés de los brasileños en diferentes áreas de la salud bucal y la estética dental. Objetivo: Este estudio utilizó la plataforma de búsqueda Google Trends® (GT) para realizar una revisión para el análisis de diez temas importantes para la odontología en Brasil. Métodos: La búsqueda se organizó con palabras claves relacionadas con la estética dental/facial (A) y la salud bucal (B). Los temas se buscaron individualmente utilizando el índice de volumen de búsqueda (IVP). Los datos cuantitativos se sometieron a una prueba de normalidad y se adoptó un índice de significancia de 0.05 . Resultados: Los principales resultados indicaron que, en el grupo A, blanqueamiento dental es el término más analizado, presentando una diferencia significativa con los demás términos: lente de contacto dental, carilla dental, armonización facial y relleno de labios, $p<0.05$. Para el grupo B: salud bucal y Herpes Bucal fueron los términos más evaluados, sin diferencia significativa entre ellos, $p>0.05$. Se destacó que hubo un IVP más alto para términos relacionados con estética dental/rostro en los últimos años (2016-2019), $p<0.05$, sin embargo el Grupo B mostró menor variación (IVP) en el mismo período. Conclusión: Hubo un aumento significativo en el uso de estética dental y facial en los últimos años en la plataforma GT y una tendencia de mantenimiento para los términos de salud bucal. Es importante que las políticas docentes en Odontología contemplen el interés de la comunidad y valoren la transmisión y sensibilización de información correcta en Odontología para el desarrollo de una buena práctica de atención clínica en la comunidad.

Descriptores: Salud Bucal; Estética; Odontología.

INTRODUÇÃO

Atualmente, diversos trabalhos de revisão sistemática (RS) têm sido publicados sobre temas de importância para a Odontologia. Estes estudos reúnem evidência científica sobre questões da prática clínica e auxiliam o 
profissional a tomar a melhor decisão para 0 atendimento do paciente ${ }^{1-3}$. O impacto destes estudos de RS está relacionado aos profissionais da área de saúde e pesquisadores das diferentes especialidades, os quais se baseiam em estudos secundários para 0 desenvolvimento de protocolos de atendimento clínico ou aperfeiçoamento das pesquisas.

Todavia, mais recentemente, começou a se verificar a importância de uma análise dos temas que a sociedade manifesta interesse nos pincipais canais digitais e bases públicas de informações, como por exemplo, o uso das plataformas Google $\AA$, Instagram $\AA$, Facebook $\AA$, YouTube $\AA^{4-6}$. A perspectiva de analisar 0 conteúdo que está sendo pesquisado pela população em bases de dados, torna-se muito relevante, uma vez que permite aos professores, profissionais e gestores envolvidos na prática e no ensino superior, direcionar ou corrigir conceitos que estão sendo divulgados na mídia.

Neste contexto, foi possível analisar o nível de interesse de usuários do Google ${ }^{\circledR}$ na América do Sul e outras regiões em relação a cárie dentária e suas manifestações, mostrando a necessidade de uma melhor conscientização da população, quanto a importância dos cuidados básicos e preventivos ${ }^{6}$. Outra pesquisa indicou que o uso de dados oriundos da internet poderia facilitar o estabelecimento de uma melhor compreensão das necessidades da população para a Odontologia, uma vez que o consumo de informações odontológicas é crescente e não necessariamente está relacionada a cárie dentária não tratada ${ }^{5}$.

As pesquisas conduzidas avaliando 0 acesso destas informações pela população são amplas. Um estudo, por exemplo, avaliou a qualidade de informações relacionadas a fluoretação de água e utilizou termos também oriundos do Google Trends® (GT), os autores indicaram que há informações relevantes, mas não adequadamente referenciadas, faltando transparência na abordagem do assunto em alguns sites ${ }^{7}$. De fato, nem sempre os conteúdos postados ocorrem de forma adequada, sendo que as informações geradas nestas bases de dados pode não apresentar um adequado nível educacional. Uma análise da plataforma YouTube® para um tema específico de ortodontia, envolvendo a pesquisa de 124 vídeos da área constatou que os vídeos estavam incompletos para uma melhor compreensão do procedimento clínico ${ }^{8}$.

Por outro lado, o uso da ferramenta GT pode atuar como um monitoramento em saúde. Um estudo recente também identificou dados de incidência da gripe Influenza na plataforma para verificação e comparação com dados do Sistema Nacional de Vigilância Sanitária da Argentina. A pesquisa apontou a utilidade benéfica no uso deste sistema para auxiliar na vigilância desta gripe ${ }^{9}$. Outra análise ampla, envolvendo diferentes países, caracterizou o interesse em informações sobre dor dentária em usuários do Google em diferentes países incluindo o Brasil. Observou-se um interesse crescente dos usuários em tópicos relacionados a dor dentária, independentemente, do país e da estação do ano, podendo as ferramentas disponíveis on-line atuarem para complementar os dados e permitir estratégias de saúde para prevenção e controle de dor dentária ${ }^{10}$.

A literatura é escassa de estudos relacionados a análise do interesse da população em aspectos de Estética Dental e Estética Facial. Portanto, a viabilização de uma pesquisa considerando diferentes palavras chaves relacionadas a estética, assim como saúde bucal é de grande relevância para o conhecimento do interesse da sociedade atual. Logo, este estudo teve como objetivo reunir dados quantitativos das principais informações acessadas pela população brasileira na plataforma GT, permitindo mapear o interesse da comunidade relacionada a estética dentária e saúde bucal nos últimos anos. A hipótese nula formulada foi a de que não haveria diferença no padrão de buscas para os diferentes unitermos avaliados nos grupos A e B.

MATERIAL E MÉTODO

\section{- Delineamento experimental}

Este estudo foi delineado conforme pesquisas publicadas, que utilizaram a plataforma Google Trends ${ }^{\circledR}$ (GT) a fim de coletar informações para comparação de variáveis em diferentes áreas ${ }^{5,6,9}$.

\section{- Amostra}

Os dados obtidos da população brasileira pela plataforma GT para avaliar estética dentária e facial (Grupo A), assim como unitermos relacionados a saúde bucal (Grupo B) foram direcionados para uma avaliação minuciosa pelos integrantes da pesquisa. A busca com dados retrospectivos foi conduzida referente aos anos de 2016 a 2020. O período escolhido foi identificado para possíveis palavras que estão atualmente sendo utilizadas no contexto da reabilitação oral e estética facial.

- Volume de dados acessados

Os dados de volume de pesquisa foram acessados a partir de agosto de 2019 para a realização da coleta de dados, com o objetivo de geração do índice de volume de pesquisa (IVP). Os respectivos unitermos utilizados são 
apresentados no quadro 1 e foram acessados para coletar a quantidade de acessos a temas relacionados aos unitermos.

Quadro 1 - Unitermos para a pesquisa na base de dados
\begin{tabular}{|c|c|}
\hline Grupo A & Grupo B \\
\hline Clareamento dental & Cárie dentária \\
\hline Lentes de contato dental & Higiene oral \\
\hline Preenchimento labial & Herpes oral \\
\hline Harmonização facial & Câncer bucal \\
\hline Faceta dental & Saúde bucal \\
\hline
\end{tabular}

Os índice de volume de pesquisas para cada unitermos foram obtidos ao longo do período e comparou-se os diferentes unitermos entre si. Neste aspecto, a base de dados Google (B atribui um score, em que um máximo valor de busca por tema e tempo relacionado gera uma pontuação de 0 a 100. Esta pontuação de acesso na base de dados também é calculada por meio de um algoritmo como descrito em estudo anterior ${ }^{5}$.

- Procedimento de coleta dos dados

A busca foi delineada considerando somente o Brasil e unitermos na língua portuguesa (Quadro 1) no período de 01/01/2016 a 13/08/2020, gerando-se um gráfico e dados em planilha para comparação do volume de pesquisa ( 0 a 100) no período considerado (4,6 anos) conforme figura 1 demonstrativa para o termo: Harmonização facial.

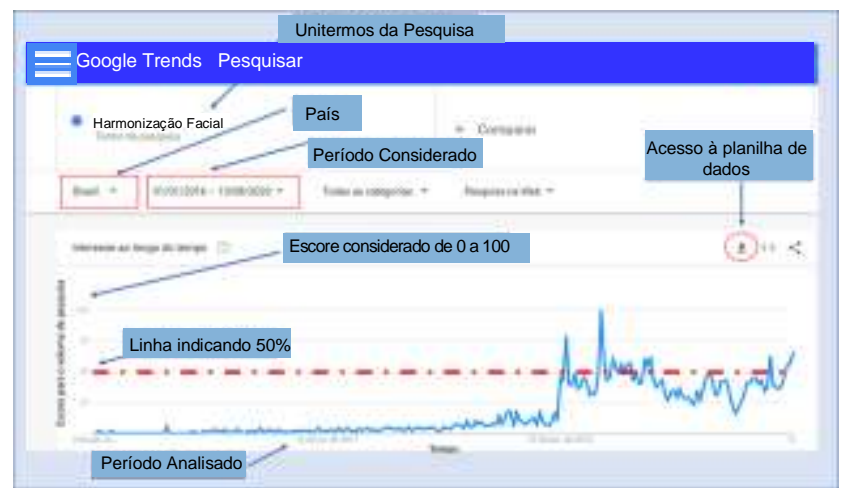

Figura 1: Procedimento utilizado para coleta de dados na Plataforma Google Trends $\AA$, exemplo utilizado para a busca da palavra Harmonização facial.

- Análise dos dados coletados e resultados

Os dados provenientes da base de dados foram organizados em tabela no formato Excel (Microsoft Office Excel, Redmond, WA, Estados Unidos) e submetidos ao software SigmaPlot (SigmaPlot, San Jose, CA, EUA) versão 12.3 e analisados em relação à distribuição normal e, posteriormente, foi adotado duas análises de variância a dois fatores (Variáveis: Grupo de unitermos e tempo) e pós teste de Tukey para análises das diferenças entre grupos, analisando-se isoladamente os grupos A (Estética facial) e grupo B (Saúde bucal).
O nível de significância considerado de 0,05 foi aplicado a todas as análises. Os resultados também foram apresentados em gráficos para comparação entre os unitermos conforme estudos anteriores da área ${ }^{6,9,10}$. RESULTADOS

$\mathrm{Na}$ comparação realizada entre os diferentes unitermos utilizados no grupo $A$, observou-se que a palavra "clareamento dental" possui índice de busca acima de $50 \%$ em quase todo o período analisado e ainda um aumento crescente no último ano. Em relação ao uso das palavras relacionadas a estética dentária e reabilitação oral com lentes de contato e faceta dental, observou-se um aumento com oscilações ao longo do período, mas também uma busca expressiva. Por fim, harmonização facial e preenchimento são unitermos com aumento mais importantes, principalmente, no último ano (2019-2020), com destaque para harmonização facial, conforme a figura 2.

Por outro lado, a análise do grupo B com unitermos relacionados a saúde bucal indicou que os unitermos cárie dentária, higiene oral, herpes oral, saúde bucal apresentaram índices com oscilações, mas sempre apresentando picos acima de $50 \%$ ao longo do período, com exceção do termo câncer bucal que apresentou algumas variações menores abaixo de $50 \%$. Os unitermos herpes oral e saúde bucal apresentaram índices com oscilações acima de $50 \%$ mais frequentemente, quando comparado aos demais, conforme figura 2 .

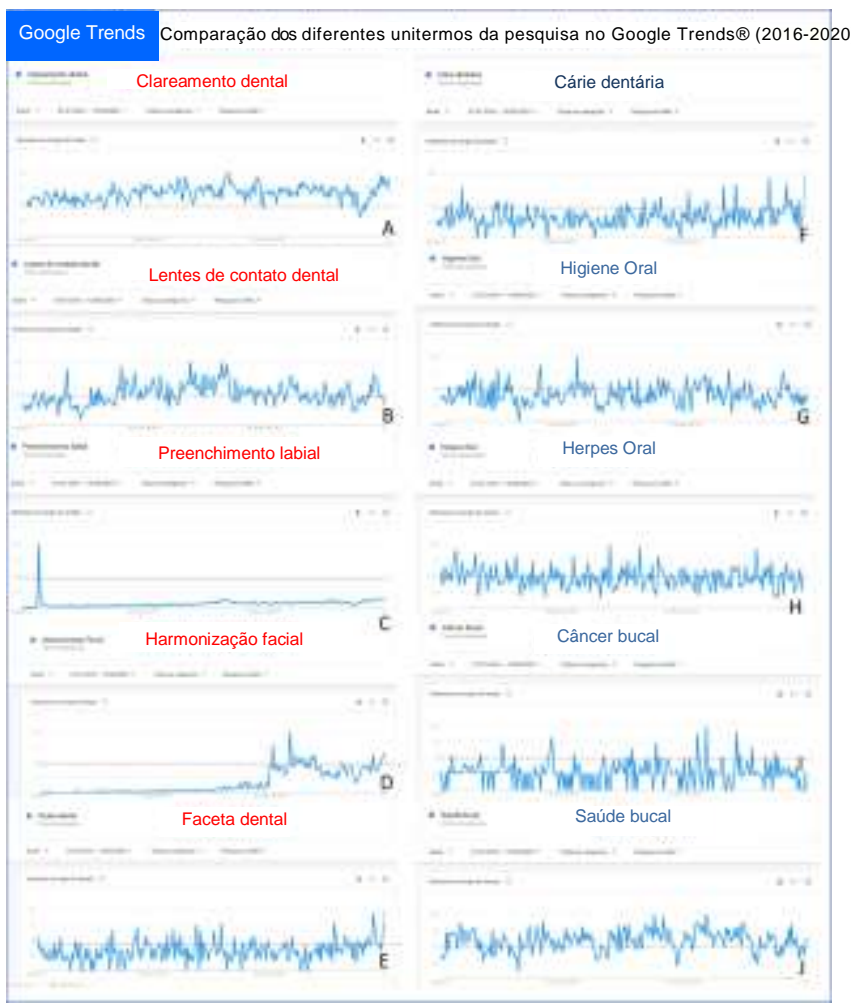

Figura 2: Unitermos utilizados na pesquisa na base de dados Google Trends $\AA^{\circledR}$ e variações no período de 2016-2020. 
Em uma busca específica das palavras relacionadas a estética facial, o unitermo clareamento dental apresenta os índices mais elevados ao longo de todo o período e apresenta diferença significativa, quando comparado aos demais $(p<0,05)$, sendo seguido pelos unitermos lente de contato dental e faceta dental. Todos os unitermos apresentaram diferença significativa entre si $(p<0,05)$, conforme figura 3.

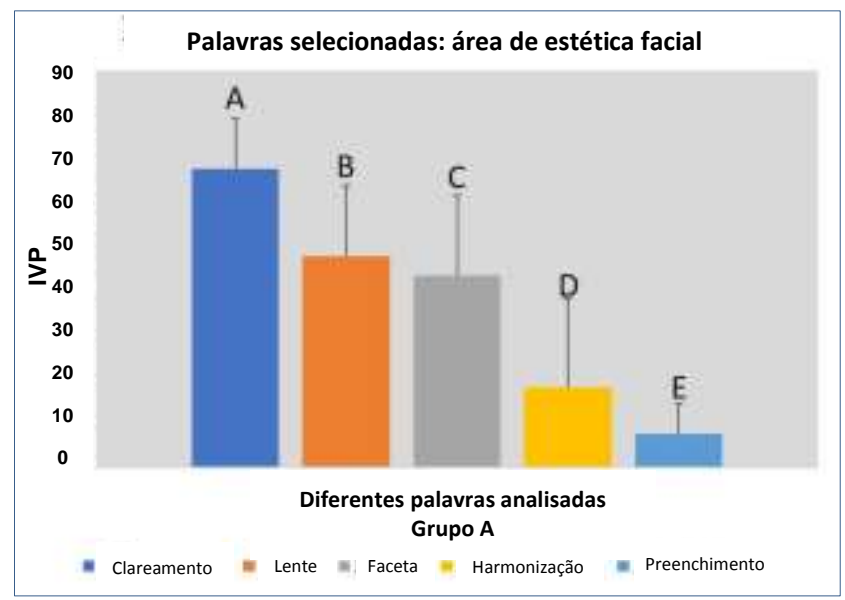

Figura 3: Análise do grupo A de palavras consideradas para estética facial e dentária. Letras diferentes maiúsculas indicaram diferença significativa $(p<0,05)$.

Em uma análise considerando os unitermos de saúde bucal, observou-se que as palavras saúde bucal e herpes oral foram os unitermos mais acessados na bases de dados e não apresentaram diferença significativa entre si $(p>0,05)$. Na sequência, observou-se as palavras higiene oral, cárie dentária e câncer bucal, sendo que estas palavras apresentaram diferença significativa na comparação com os demais grupos $(p<0,05)$, conforme figura 4 .

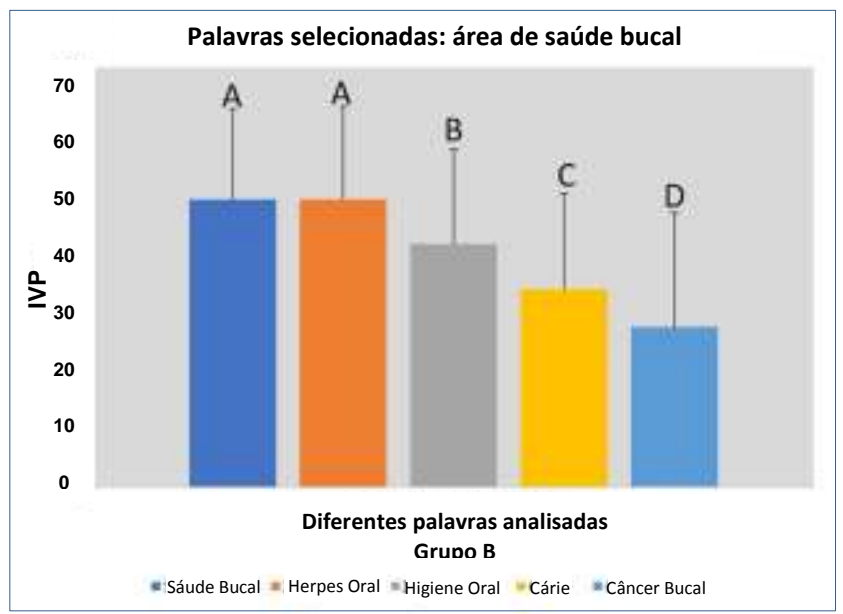

Figura 4:- Análise do grupo $\mathrm{B}$ de palavras consideradas para saúde bucal. Letras maiúsculas diferentes indicaram diferença significativa $(p<0,05)$, letras iguais maiúsculas indicaram que não houve diferença significativa $(p>0,05: A, A)$.

Considerando os diferentes anos avaliados, observou-se que para o grupo $A$ houve um aumento considerável entre os diferentes anos em todos os períodos considerados, sendo que houve diferença significativa na comparação entre todos os anos $(2016,2017,2018$ - $p<0,05)$, com exceção na comparação de 2019 e 2020 (p>0,05), demonstrando um aumento ao longo dos anos e estabilização no último período, conforme figura 5. Por outro lado, no grupo $B$ houve oscilações no período, com queda em 2017 e aumento até 2019, inclusive somente houve diferença significativa na comparação entre os anos de 2017 e 2019 ( $p<0,05)$, posteriormente, com redução novamente no IVP, demonstrando um equilíbrio para os tópicos, conforme figura 5.

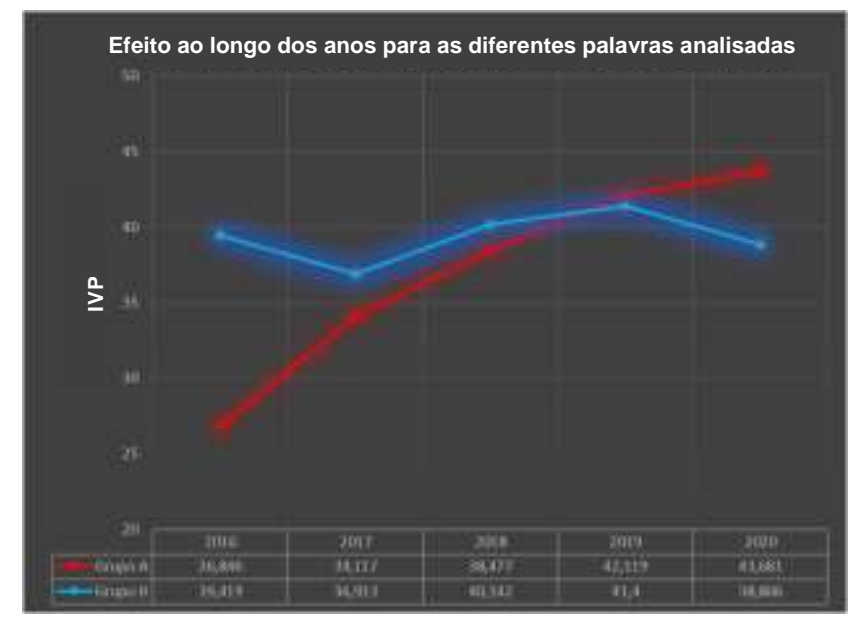

Figura 5: Análise do tempo em cada grupo de palavras. Grupo A todos os anos apresentaram diferença significativa entre $s i$ $(p<0,05)$ com exceção na comparação de 2019 e 2020 ( $p>0,05)$. No grupo $B$ somente houve diferença significativa na comparação de 2017 vs. $2019(p<0,05)$, os demais anos não apresentaram diferença significativa $(p>0,05)$.

DISCUSSÃO

A hipótese nula formulada sobre a similaridade no padrão das buscas relacionadas aos dois grupos de palavras (saúde bucal e estética dentária e facial) foi recusada, uma vez que houve diferenças ao se analisar o perfil das buscas nos dois diferentes grupos considerando os anos avaliados.

De uma forma geral, as palavras relacionadas a saúde bucal indicaram uma predominância de busca neste período com um IVP acima de $50 \%$, isto tem demonstrado um resultado favorável para a busca destes assuntos no Brasil e, já foi verificado em pesquisa anterior sobre a influência dos termos "cárie dentária" e "dor dentária" na América do Sul e diferentes países ${ }^{6}$. Em um estudo amplo que analisou a plataforma GT de 2004 a 2017, Cruvinel et al. ${ }^{6}$ observaram a possibilidade de redução no interesse das buscas em situações em que poderia existir uma demanda estável de conhecimento sobre saúde bucal. Além disso, foi relatado que há diversas variáveis que podem interagir na resposta da busca destes itens relacionados a saúde bucal como o acesso à informação, nível de educação das 
pessoas regionalmente e o conhecimento prévio em saúde bucal. Isto pode explicar variações e algumas reduções no padrão de busca observadas nos diferentes anos avaliados.

Os unitermos escolhidos para o grupo de palavras em saúde bucal, mostraram aspectos importantes de buscas usuais da comunidade. Semelhantemente, em uma estudo recentemente realizado, o unitermo "câncer oral" foi avaliado na plataforma GT indicando uma relevância mundial nas pesquisas realizadas. Os autores indicaram também a existência de outros padrões de buscas para "câncer de língua", "úlcera de boca", "sinais e sintomas" . Apesar de ter sido realizado em contexto mundial, Patthi et al. ${ }^{4}$, avaliaram de 2004 a 2016 vários unitermos no GT e indicaram que o termo "gengivite" foi a palavra com maior busca no período, o que mostra importância do conteúdo em saúde bucal.

Destacou-se em nossa pesquisa os unitermos de saúde bucal e herpes oral como os principais unitermos neste grupo de palavras, de uma forma geral, saúde bucal relaciona-se com grande parte das buscas realizadas e indica um papel positivo no controle e melhoria da saúde pelo indivíduo, já a manutenção de IVP elevado para o unitermo "herpes oral" também demonstrou uma preocupação da comunidade, uma vez que dados da Federação Médica Brasileira indicaram que até $95 \%$ da população brasileira podem apresentar herpes oral ${ }^{11}$, isto reforça a importância de medidas educativas nesta área buscando difundir informações corretas de diagnóstico e tratamento.

Nesta pesquisa foram observadas alterações conforme o decorrer dos meses para as palavras pesquisadas. Este efeito de sazonalidade é justificado conforme o período do ano, podendo existir uma influência dos períodos de férias, ano letivo escolar, mudanças de rotina nos hábitos, isto justifica alterações que ocorrem ao longo dos períodos avaliados $^{5,6,10}$. Estas mudanças de sazonalidade já foram demonstradas em outros tipos de estudos clínicos na literatura ${ }^{12}$.

Por outro lado, pode ser que a sazonalidade não tenha o mesmo efeito na área de estética uma vez que a busca pelo interesse pode se comportar de uma forma diferente. Estes dados demonstraram uma padrão crescente para algumas buscas retratado pelo IVP, estas informações estão concordantes com outra pesquisa na área de cirurgia de implantes dentários e cirurgia estética facial, o qual relatou ampliação do interesse ao longo dos últimos anos ${ }^{13}$.

De fato, os unitermos na área de estética dentária e facial tiveram um comportamento diferente. A busca por palavras-chave na área de estética dentária tem indicado para algumas áreas uma elevada procura, como em clareamento dentário ou em outras áreas em desenvolvimento mais recentemente. Isto está de acordo com pesquisa recentemente publicada, que indica um papel mais atual da comunidade em buscar procedimentos estéticos minimamente invasivos $^{13}$. No Brasil, há um aumento na busca pelo termo clareamento dentário. Isto é justificado por meio de matérias na mídia que retratam e abordam a busca por esta modalidade de tratamento estético, indicando um aumento de procura em até $30 \%$ em $2019^{14}$. O grande interesse da população pelo tema, fez as indústrias aumentarem a oferta por produtos de autoaplicação, com a promessa de resultados similares aos obtidos pelos cirurgiões-dentistas a um preço mais acessível ${ }^{15}$. Esta informação mostra a relevância de uma adequada conscientização da comunidade, da grande importância da realização desse procedimento apenas com o acompanhamento e prescrição clínica por profissionais habilitados. Ainda neste aspecto, as demais palavras relacionadas a reabilitação oral como facetas e lentes de contato tem ampliado o interesse na comunidade, porém não semelhantemente, ao clareamento dentário. Isto pode ser inerente ao desconhecimento da sociedade em relação a termos utilizados na busca. Todavia, em relação a palavra harmonização e preenchimento, observamos um aumento de IVP recentemente. Em relação a harmonização facial é perceptível o aumento no último ano de2019, isto também vem de encontro com a aprovação como especialidade pelo Conselho Federal de Odontologia, o qual reconheceu esta área como uma especialidade odontológica na resolução CFO 198/2019 ${ }^{16}$. Neste aspecto o CFO lançou uma medida nova para especificar as indicações e possibilidade de atuação do profissional especialista CFO $230 / 2020^{17}$. Torna-se, assim, muito importante que as instituições de ensino superior em Odontologia possam capacitar da melhor forma possível 0 profissional, que irá realizar o procedimento e diante de uma maior busca de informações sobre o tema, também realça o impacto na comunidade pela busca na área de estética facial.

Há limitações neste estudo conforme já 
relatado na literatura, os dados são oriundos de usuários da plataforma Google com um algoritmo único., Em outras plataformas de busca não foi realizada uma comparação, assim como os dados obtidos retratam informações específicas de usuários com acesso à internet neste período avaliado., É importante destacar que buscas podem ser duplicadas pelo mesmo usuário ou resultados podem ser ampliados devido a campanhas de saúde e mídia regional ${ }^{5,18,19}$. Outro ponto importante relaciona-se à amostra avaliada, é difícil analisar a amostragem correta da busca realizada e determinar se os dados oriundos estão contemplando adequadamente diferentes subgrupos ${ }^{4,13}$. Os unitermos escolhidos também podem refletir uma limitação, principalmente, na área de estética, porque ainda são de domínio de pessoas com acesso a informação de campanhas de saúde e estética dentária. Estudos mais amplos envolvendo diferentes unitermos, palavras usuais a comunidade e com dados específicos para estados e regiões do Brasil devem ser realizados nos próximos anos.

Por fim, os estudos na área considerando o uso de GT são relevantes uma vez que contribuem para avaliação do padrão de busca de informações nesta importante plataforma, assim como interesses de variações regionais e sazonais, permite também um adequado estudo de doenças, procedimentos em saúde que podem estar aumentando a demanda por busca de informações na internet ${ }^{4,5,13,20}$.

CONCLUSÃO

Com base na metodologia empregada e nos resultados obtidos concluiu-se que 0 unitermo com maior índice de procura foi "clareamento dentário". Dentro deste grupo na área de estética dentária e facial, também se destacou o aumento crescente no último ano de harmonização facial. No contexto geral, este grupo de palavras foi o que apresentou o maior crescimento nos últimos anos. A busca pelos unitermos em saúde bucal demonstrou um equilíbrio entre as diferentes palavras com maior destaque para saúde bucal e herpes oral. Porém, estudos mais amplos devem ser realizados avaliando diferentes unitermos, palavras usuais da comunidade e considerando as diferentes características de regiões e sazonalidade.

REFERÊNCIAS

1. Lopes LFdP, da Silva VF, Santiago JF, Jr., Panzarini SR, Pellizzer EP. Placement of dental implants in the maxillary tuberosity: a systematic review. Int J Oral Maxillofac Surg. 2015 2015;44(2):229-38.

2. Santiago Junior JF, Biguetti CC, Matsumoto MA, Kudo GAH, Silva RBP, Saraiva PP et al. Can Genetic Factors Compromise the Success of Dental Implants? A Systematic Review and Meta-Analysis. Genes (Basel). 2018;9(9):444.

3. Medeiros FCFL, Kudo GAH, Leme BG, Saraiva PP, Verri FR, Honório HM et al. Dental implants in patients with osteoporosis: a systematic review with meta-analysis. Int $\mathrm{J}$ Oral Maxillofac Surg. 2018;47(4):480-91.

4. Patthi B, Kumar JK, Singla A, Gupta R, Prasad M, Ali I et al. Global Search Trends of Oral Problems using Google Trends from 2004 to 2016: An Exploratory Analysis. J Clin Diagn Res. 2017;11(9):ZC12-16.

5. Aguirre PE, Coelho $M$, Oliveira $T$, Rios $D$, Cruvinel AF, Cruvinel T. What Can Google Inform Us about People's Interests regarding Dental Caries in Different Populations? Caries Res. 2018;52(3):177-188.

6. Cruvinel T, Ayala Aguirre PE, Lotto M, Marchini Oliveira T, Rios D, Pereira Cruvinel AF. Digital behavior surveillance: Monitoring dental caries and toothache interests of Google users from developing countries. Oral Dis.2018;25(1):339-47.

7. Frangos Z, Steffens M, Leask J. Water fluoridation and the quality of information available online. Int Dent J. 2018;68(4):253-61.

8. Lena $Y$, Dindaroğlu F. Lingual orthodontic treatment: A YouTube ${ }^{\mathrm{TM}}$ video analysis. Angle Orthod. 2018;88(2):208-14.

9. Orellano PW, Reynoso JI, Antman J, Argibay O. Uso de la herramienta Google Trends para estimar la incidencia de enfermedades tipo influenza en Argentina. Cad Saúde Pública. 2015;34(4):691-700.

10. Lotto M, Ayala Aguirre P, Rios D, Andrade Moreira Machado M, Pereira Cruvinel A, Cruvinel T. Analysis of the interests of Google users on toothache information. PLoS One. 2017;19(12):e0186059.

11. Brasileira FM. Herpes acomete $95 \%$ da população brasileira. Federação Médica Brasileira. Acesso 03 de setembro de 2020.

12. Jones AG, McDonald TJ, Hattersley AT, Shields BM. Effect of the holiday season in patients with diabetes: glycemia and lipids increase postholiday, but the effect is small and transient. Diabetes Care. 2014;37(5):e98-9.

13. Shen JK, Every J, Morrison SD, Massenburg BB, Egbert MA, Susarla SM. Global Interest in Oral and Maxillofacial Surgery: Analysis of Google Trends Data. J Oral Maxillofac Surg. 2020;78(9):1484-91.

14. Interplan M. Clareamento dental se destaca entre os procedimentos estéticos no país. Globo. Accessed 03/09/2020, 2020. https://g1.globo.com/sp/presidente-prudenteregiao/especial-publicitario/interplan- 
assistencia-funeral/interplan-ao-seu-lado-emtodos-os-

momentos/noticia/2019/09/03/clareamentodental-se-destaca-entre-os-procedimentosesteticos-no-pais.ghtml.

15. Kym, HA, Kim JW, Kim SJ. Double-blind Randomized Study to Evaluate the Safety and Efficacy of Over-the-counter Tooth-whitening Agents Containing 2.9\% Hydrogen Peroxide. Oper Dent. 2018;43(3):272-81.

16. Odontologia CFd. Resolução CFO-198, de 29 de janeiro de 2019. Conselho Federal de Odontologia Accessed 03/09/2020, 2020. http://sistemas.cfo.org.br/visualizar/atos/RESOL U\%C3\%87\%C3\%83O/SEC/2019/198.

17. Odontologia CFd. RESOLUÇÃO CFO-230, de 14 de agosto de 2020. Conselho Federal de Odontologia. Accessed 03/09/2020, 2020. http://sistemas.cfo.org.br/visualizar/atos/RESOL U\%c3\%87\%c3\%83O/SEC/2020/230.

18. DeRouen TA. Promises and Pitfalls in the Use of "Big Data" for Clinical Research. J Dent Res. 2015:107S-9S.

19. Metcalfe D, Price C, Powell J. Media coverage and public reaction to a celebrity cancer diagnosis. J Public Health (Oxf). 2011;33(1): 80-5.

20. Carneiro HA, Mylonakis E. Google trends: a web-based tool for real-time surveillance of disease outbreaks. Clin Infect Dis. 2009; 49(10):1557-64.

\section{CONFLITO DE INTERESSES}

Os autores declaram não haver conflitos de interesse

\section{AUTOR PARA CORRESPONDÊNCIA}

\section{Prof. Dr. Joel Ferreira Santiago Junior}

Centro de Ciências da Saúde,

Centro Universitário Sagrado Coração - Unisagrado USC,

Rua: Irmã Arminda, 10-50, Jardim Brasil, 17011-160 Bauru - SP, Brasil

Tel.: +55 142107 7374. Fax: +55 21077206

E-mail:joel.junior@unisagrado.edu.br 